\title{
A CHANGE OF PROFILE BASED ON LOCATION
}

\author{
Suryabhan Gupta ${ }^{1}$, Amol Joglekar ${ }^{2}$ \\ ${ }^{1}$ M.Sc Computer Science student, Mithibai College, Mumbai, Maharashtra, India \\ ${ }^{2}$ Guide and Professor, Computer Science Mithibai College, Mumbai ,Maharashtra, India
}

\begin{abstract}
In today's world time management is a big task. There could be the possibility that we may forget to do some important and urgent jobs which could results in a negative way for us. Also we have a trend of using smart phones supporting different tools and applications which could make our life simple. Today devices and phones run on many different types of operating systems such as IOS, Android etc. The proposed study is based on android based devices such as smart phones and tablets running on the Android operating system, since Android is designed to be an open source OS it gives a wide opportunity to any developer to develop and try different applications on such devices. In the proposed system we will discuss about the application which provides the service of automatic profile changing based on the user's location from one to other (e.g. from normal to work mode or vice versa). It uses internet connection and GPS services to learn about the user's current location and accordingly it updates the profile.
\end{abstract}

Keywords_LBS, anroid OS, profile, GPS.

\section{INTRODUCTION}

This proposal is made on the basis of "Location based services on smart phone using Android application." and is been designed to be automatic using the GPS services provided by the smart phone manufacturers and using the internet services on the smart phone. The motivation is that to provide the android smart phone user with an application that does not bother him/her to change the profile of their phone manually each time they reach to a certain place, but rather to let them set a preferred profile for that place and it would be updated automatically as soon as the user reaches that place, and also when he leaves that place the profile will again be updated to the default or normal mode. This can be possible because of the rapid growth of the wireless communication systems and global location positioning systems. We know that the Android is an open source platform it allows anyone to try and develop application for it and use it for the specific function on the devices running Android. Since the mobile devices are built having limited power and storage facility, it is ideal to build applications for one specific purpose or one specific work. In this proposed system we have designed an application that will provide the automatic profile change. There are other various location based services as well that could be implemented on the android devices. However there are some limitations to the frequent responses of these applications depending on the goodness of internet connections and also in the GPS technology's accurate functioning.

The various location based services that are available are

1) Automatic profile updater for Android phone

2) Location tracking of a person by others

3) Nearest friend finder etc

\subsection{Location based Services}

A location-based service (LBS) is a mobile application that is dependent on the location of a mobile device, like mobile phone. Virrantaus et al [1] defined LBS services as follows:

"Information services accessible with mobile devices through the mobile network and utilizing the ability to make use of the location of the mobile device"

Open Geospatial Consortium [2] defined "A wireless-IP service that uses geographic information to serve a mobile user, any application service that exploits the position of a mobile terminal."

A Location Based Service (LBS) is an information and entertainment service, accessible with mobile devices through the mobile network and utilizing this ability to make use of geographical position of the mobile device. LBS services can be used in a variety of way, such as work, health, personal life, etc. LBS include services to identify the location of a person or object, such as discovering the nearest ATM or the nearest filling station, tracking location of friend or employee. LBS services include order tracking and vehicle tracking services.

There are two major parts-

1) Obtaining the current location of the user

2) Utilize the obtained information to provide the user a specific service

\subsection{Android Operating System}

Android delivers a complete set of software for mobile devices: an operating system, middleware and key mobile applications. Android was built from the ground-up to enable developers to create compelling mobile applications that take full advantage of all a handset has to offer. It was built to be 
truly open. Android is built on the open Linux Kernel. Android support LBS Application Programming Interfaces (APIs) . Location service allows finding out the device current location. The application can request for periodic update of the device location information. The application can also register a intent receiver for proximity alerts like when the device is entering and existing from an area of given longitude, latitude and radius.

On a basic level, Android is a distribution of Linux that includes a Java Virtual Machine (JVM), with Java being the preferred programming language for most Android applications. The Android Software Development Kit (SDK) includes a debugger, libraries, a handset emulator, documentation, sample code and tutorials. Android's official integrated development environment is Eclipse using the Android Development Tools (ADT) plug-in. SQLite database support is integrated into the Android platform. The ADT plugin includes an Android emulator that allows for the simulation of GPS and Wi-Fi. The Android emulator is depicted

\subsection{Android Location API}

These are the different classes present under Location API package to retrieve the Location information of the user.

Location Manager- The class provides access to the location service. It also provides facility to get the best Location Provider as per the criteria.

Location Provider- It's an abstract super class for location providers. A location provider provides periodic reports on the geographical location of the device.

Location Listener- This class provides callback methods which are called when location gets changed. The listener object has to be registered with the

Location Manager - The class provides the application to choose suitable Location Provider by providing access to set of required properties of the Location Provider. Android also provide an API to access the Google maps. So with the help of the Google maps and the location APIs the application can show required places to the user on the map.

\subsection{Google Places API}

On 10 May, 2011, at the Google I/O developer Conference in San Francisco, Google announced the opening up and general availability of the Google Places API. The Google Places API is a service that returns data about Places - defined within this Web Service as, spatial locations, or preferred points of interest - using HTTP requests. Place response specifies locations as latitude/longitude coordinates. The four types of requests are available with the Google Places API There are 4 fundamental Place services available:

$\square$ Place Searches - It returns an array of nearby Places based on a location defined by the user.

$\square$ Place Details - It returns more specific data about a user defined Place.

$\square$ Place Check-ins - It allows the request that a person has checked in to a Place. $\square$ Place Reports - It allows the users to add new locations to the Place service, and to delete Places that the application has added to the database.

\section{GPS TECHNOLOGY AND ITS WORKING}

In the recent years there has been a drastic increase in the usage of smart phones by people specially those running on the android operating system. These smart phones are now coming lashed with GPS (Global Positioning System) inside of them to update the location of the phone anywhere in the world. Previously for the same reason the cell IDs were being used. But in the current era that has been replaced by the GPS technology. GPS works on the basis of calculating the latitude and the longitude and according to which after doing some mathematical calculations the exact location of the phone is determined. Nowadays since GPS is integrated with the smart phones a wide variety of location based services and applications are available to be used and are being used on a large scale by smart phone users.

Also there is a need of an application which is also location based and uses the GPS and data services of the phone to provide a specific service to the user. It also consists of a middle layer of software as well hardware to get access to the basic features of LBS(location Based Services) such as tracking location and collecting location information and providing a consistent interfacing to the LBS applications.

It also consists of many components which are known as LBS components and are shown on the figure below.

It has the parts as shown in the figure below foe the particular uses of them as their name suggests like LBS application is the standalone application built for a specific functionality, smartphones are the devices on which this application is installed and run these phones has built in sensors for location tracking, it also uses a server to exchange the information, middleware is used to provide an interface between the mobile phone and the application to run it, location tracking involves the GPS data and GIS data synchronization, finally the LCS service collects the location of the device.

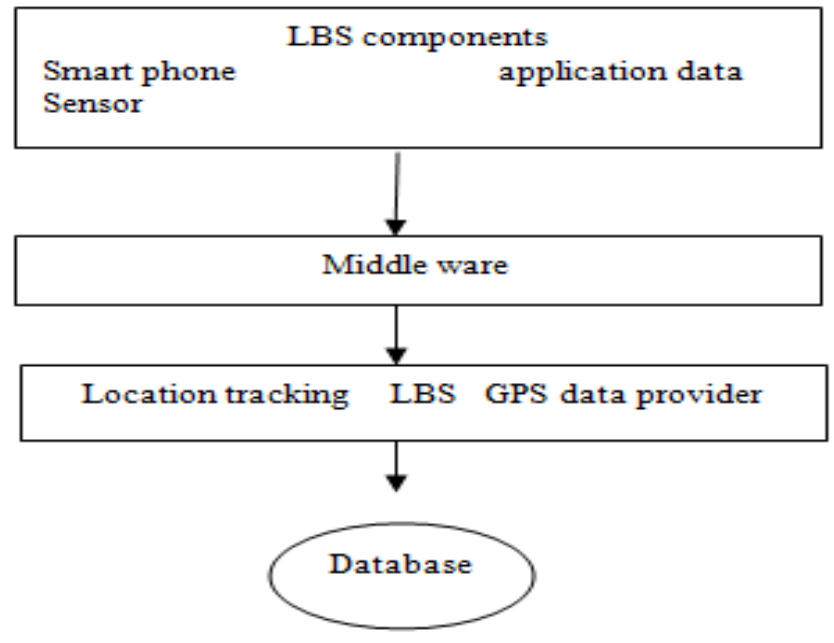

Fig 1: architecture of LBS 


\section{LITERATURE SURVEY}

Prof. Seema Vanjire, Unmesh Kanchan, Ganesh Shitole, and Pradnyesh Patil[3] states that the location based service is beneficial in a variety of ways using the LBS components and GPS trackers available in the phones. They suggested that the android applications can be merged with google maps APIs and using that advantage they can provide services like "location tracking of person", "nearest friend finder", "profile Changer based on the location" and so on. They also revealed in their study that the location of a device is tracked in many ways such as using the mobile's IDs, using the satellites which include calculating the differences in the time of a signal reaching to the device from a particular satellite. They said that there are 24 satellites in the earth's orbit and they optimize the location of the device between the radius of 5 to 10 kilometers accuracy. This is the GPS technology. Whereas the GSM based ID using location tracker gives a low accuracy of the device being anywhere in the radius of 150 meters with a granularity. It also focuses on the reputation based security.

Vedang Moholkar, Prathamesh Hule, Mandar Khule, and Sumit Sourabh[4] suggested location based automatic service providing for the smartphone users. They focused on developing a application that provide profile changing, friends location and also remind the person whom the user is going to meet at a certain place if both of them have the similar applications installed on their devices. This will save a lot of time for both and also the headache of waiting for the other to come will be taken care of by the application.

Sandeep Kumar, Mohammed Abdul Qadeer, and Archana Gupta[5]advocated about building application which will provide the information of the geographic locations to the users who are touring abroad or in their own country and are not aware of certain places ,their suggestion indicates that if such application is build then there will be no need of hiring a tour guide for taking you tours around the country an even around the world. This will be made possible by the application built as per their suggestions. This will have information about the hotels, filling stations, tour locations and also will use the GPS technology to guide the way of the user to the selected location.

Pankti Doshi, Pooja Jain, and Abhishek Shakwala[6] focused on the similar things of providing similar services of locating gas filling stations and nearest hospitals etc using the mobile phone application built for the purpose. They also suggested the integration of the Google Maps APIs in the application to fetch the location in a more clear and more understandable format. There are a variety of Goggle maps APIs are available such as Grid View, satellite view, map view and so on. So the particular integration of the Map will help the user see the location clearly from the place where they are, and also the route will be navigated to them with the help of the Google Maps. They have also suggested its various usage fields too.

Amit Kushwaha, and Vineet Kushwaha[7] stated about the Different (LBS) components and about the architecture of these components and how they work together as one integrated system with Android application and Google map, GPS integration in an application to provide various location based services to the users, such as emergency services , health hazards and tracking the location of a patient and sending an ambulance for the emergency services etc.

\section{RESEARCH METHODOLOGY}

\subsection{Proposed System}

Proposed system is also a location based service provider application in which it provides the user the choice to select the location and set a specific profile for that location according to his own choice and keep the internet services and the GPS services on in his phone. So as per his/ser preference the profile of the phone will automatically change by fetching the correct location of the user set by the user and again to set the normal mode when the user moves away from that location. It has some inbuilt profiles to let the user either choose one of them or even they can create the profile of their own choice for the particular place and set it to be activated and deactivated accordingly. Here we proposing to try and implement the idea of building a location based application and integrating the Google Map APIs in it to provide the most possible accurate results to the user about a particular location and also the proposal is to make the application work in a particular location and keep the profile selected for that location to be active until the user moves away from that location. Once the user moves away from that location the application will run again and update the profile to the normal or default mode from the one activated for the location (may be silent).

\subsection{Flow Chart}

The proposed System is Depicted By the following flow chart shown in figure 2 below.

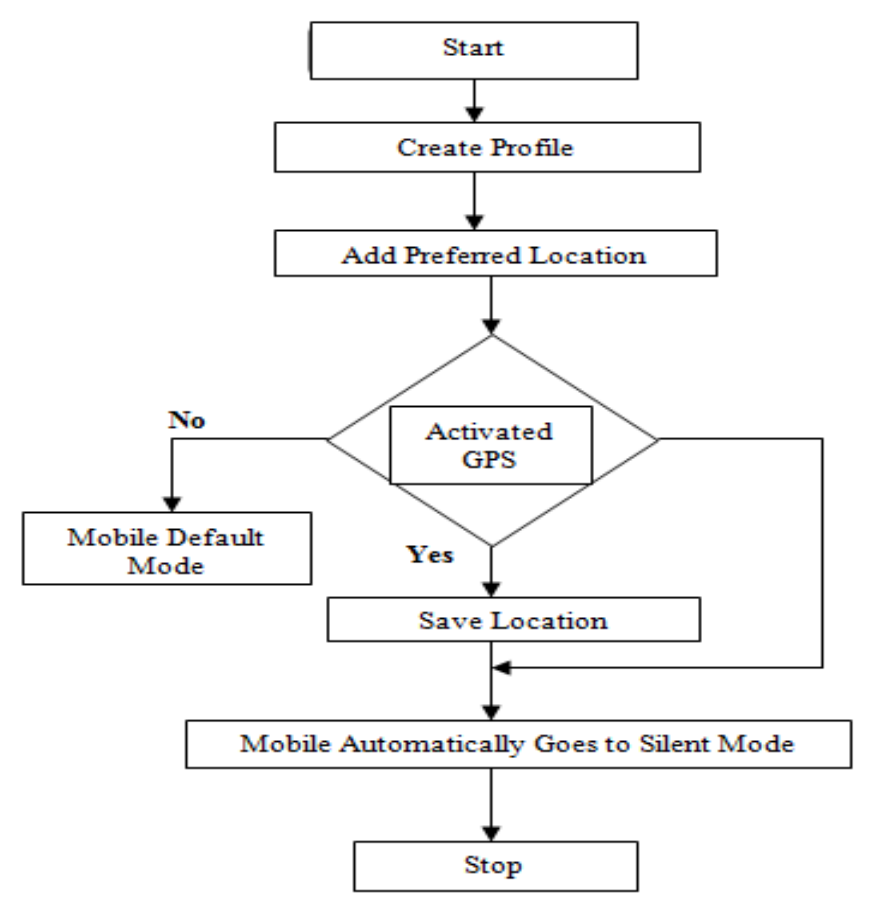




\subsection{TestData}

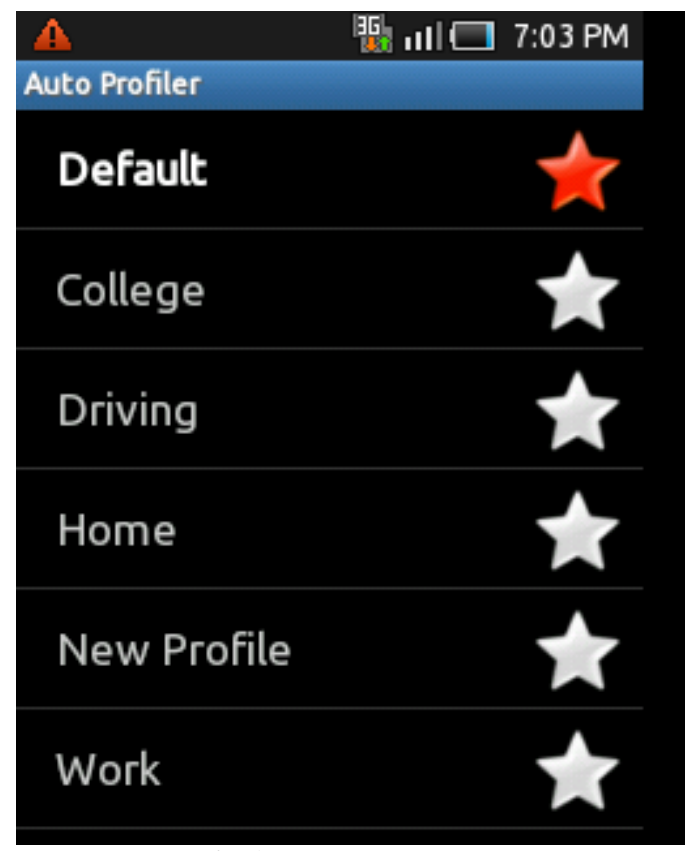

Fig 2: GUI interfacing

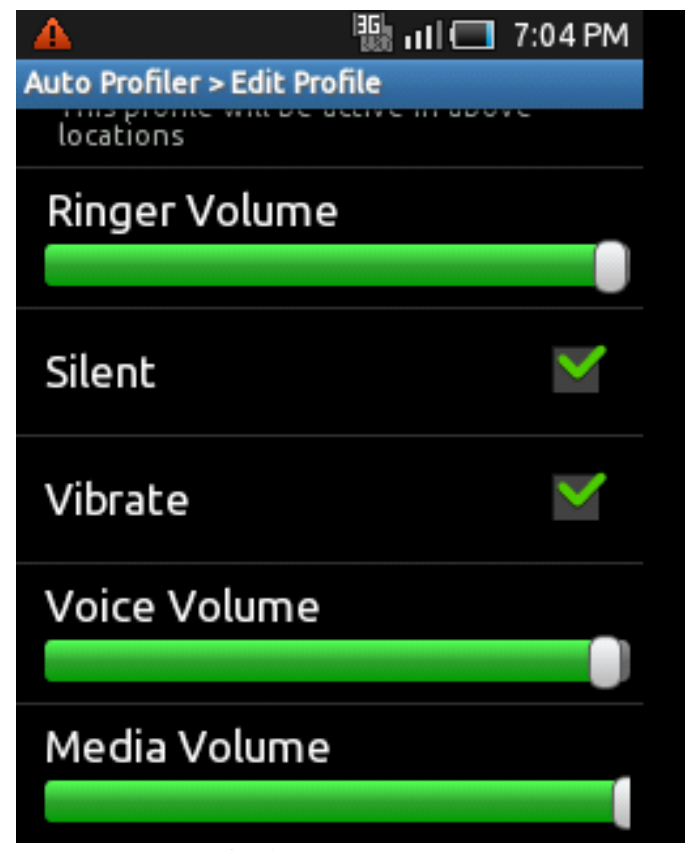

Fig 3: profile setting

\section{FUTURE SCOPE}

The Auto-Profiler is an Android application that features an automatic sound profile silencing feature and a dynamic alarm system. The dynamic alarms and automatic phone silencing system will be based on GPS. The phone will automatically change the sound profile to silent or vibrate when it is close to a building. The user will be able to add alarms to the application by entering Course Reference Numbers (CRN) which will automatically add the required information such as class location and meeting times. AutoProfiler will synchronize with Google Calendar.
Our system provides manual and automatic modes to change the profiles. In automatic mode, our application sets a profile of mobile phone automatically according to predefined location. When there will be no locations provided for any profile then "Default" profile gets activated. An application provides various ringing settings for single profile like silent mode, voice volume, media volume and ringing volume. We can set number of locations for single profile.

The model can be implemented using different languages and can be made more reliable and fast. Some extra features can be added like alerts, friend locator etc.

\section{CONCLUSION}

Auto profiler is an excellent tool that helps us in our daily life. We might forget to mute our phone before entering into any important meeting and lose the impression of the delegates sometimes. So using this application the user can focus on his daily activities without worrying to monitor his mobile device. Once he sets all his profiles according to the schedule the application takes care of it and changes the profiles according to the Location. Also, the application itself will change the profile to the normal or the default mode once the user leaves that location. It also allows the user to create new profiles based on the locations he will be going to and to also manipulate the already existing profiles without any bondage. The behavior of the model is totally depend on internet connection or Wi-Fi networks.

\section{REFERENCES}

[1]. Virrantaus, K., Markkula, J., Garmash, A., Terziyan, V., Veijalainen, J., Katanosov, A., and Tirri, H. "Developing gissupported location-based services". In Web Information Systems Engineering (2001), IEEE, pp. 66_75.

[2]. Consortium, O. G. Open location services 1.1, 2005.

[3]. Prof. Seema Vanjire, Unmesh Kanchan, Ganesh Shitole, and Pradnyesh Patil, "Location Based Services On Smartphone Trough Android Application" in International Journal of Advanced Research in Computer and Communication Engineering Vol. 3, Issue 1, January 2014, ISSN (Online) : 2278-1021.

[4]. Vedang Moholkar, Prathamesh Hule, Mandar Khule, and Sumit Sourabh, "Automated Location Based Services" Volume 4, Issue 2, February 2014, ISSN: 2277 128X, in International Journal of Advanced Research in Computer Science and Software Engineering.

[5]. Sandeep Kumar, Mohammed Abdul Qadeer, and Archana Gupta, "Location Based Services Using Android" in Aligarh Muslim University, Aligarh 202002, India, 2009 IEEE.

[6]. Pankti Doshi, Pooja Jain, and Abhishek Shakwala, “" Location Based Services And Integration Of Google Maps In Android" in International Journal Of Engineering And Computer Science ISSN:2319-7242, Volume 3 Issue 3 March,2014 Page No. 5072-5077.

[7]. Amit Kushwaha, and Vineet Kushwaha in "Location Based Services Using Android Mobile Operating System" in International Journal of Advances in Engineering \& Technology, Mar 2011, ISSN: 2231-1963. 
[8]. www.IJCSI.org

[9]. Chris Haseman, Android Essentials, firstPress, 9_17, 2008

[10].http://developer.android.com/guide/topics/fundamentals.

html

[11]. Google Directions API

https://developers.google.com/maps/documentation/direction

s/

[12]. Google Places API

https://developers.google.com/places/documentation/ 